\title{
Modelando trajetórias de aprendizagem utilizando princípios de design baseado em blocos: um estudo de caso aplicado à aprendizagem em desenvolvimento web
}

\author{
Paulo Santana Rocha - UFRGS/PPGIE, rochap01@ gmail.com \\ José Valdeni de Lima - UFRGS/PPGIE, valdeni@inf.ufrgs.br \\ Rafaela Jardim - UFRGS/PPGIE, rafa.rjardim@gmail.com \\ Dauster Souza Pereira - UFRGS/PPGIE, daustersp@gmail.com \\ Igor Kuhn - UFRGS/PPGIE, kuhnigor@gmail.com
}

Roseane de Lima Parente Rocha - FADERGS, roseparenterocha@gmail.com

\begin{abstract}
Resumo: Esta pesquisa apresenta uma proposta de modelo gráfico para representar trajetórias de aprendizagem utilizando os princípios de design das linguagens de programação visuais. Tal abordagem permite, através da relação entre conceitos, modelar percursos pedagógicos complexos, sugerindo um instrumento promissor no sentido de garantir uma visão macro eficiente das trajetórias. Além disso, um estudo de caso foi realizado no curso de Informática da Universidade Federal do Rio Grande do Sul, onde 27 estudantes foram convidados a estruturar trajetórias de aprendizagem utilizando o modelo e ferramentas propostos nessa pesquisa. Como resultado foi possível observar a geração de 50 representações de trajetos, com abordagens distintas para assuntos inerentes à área de desenvolvimento para internet. Não obstante, os dados quantitativos de desempenho evidenciam que, ao estruturar as trajetórias na forma de blocos, ocorre ganho quanto à assimilação dos conceitos, além de uma visível evolução do aspecto da homogeneidade da turma.
\end{abstract}

Palavras-Chave: trajetórias de aprendizagem; programação visual; modelagem.

\section{Modeling hypothetical learning trajectories using principles of block-based design: a case study applied to learning in web development}

Abstract: This research presents a proposal of a graphic model to represent learning trajectories using the design principles of visual programming languages. This approach allows, through the relationship between concepts, to model complex pedagogical paths, suggesting a promising instrument in order to guarantee an efficient macro vision of the trajectories. In addition, a case study was carried out in the Informatics course of the Federal University of Rio Grande do Sul, where 27 students were invited to structure learning trajectories using the model and tools proposed in this research. As a result, it was possible to observe the generation of 50 route representations, with different approaches for subjects inherent in the area of development for the internet. Nevertheless, the quantitative performance data show that, when structuring the trajectories in the form of blocks, gains in the assimilation of the concepts, as well as a visible evolution of the homogeneity aspect of the class.

Keywords: learning trajectories; visual programming; modeling.

\section{Introdução}


Segundo (BORBA, 2016) é crescente na literatura pesquisas relacionadas ao estudo de tecnologias educacionais, sobretudo em aspectos ligados à transformação das práticas educativas, normalmente vinculadas à contextos onde sua utilização pode apoiar o processo ensino e aprendizagem.

Neste sentido, o estudo das trajetórias de aprendizagem é recorrente na literatura, onde para esta pesquisa considera-se que uma trajetória é um caminho através de um corredor conceitual, onde existem obstáculos previsíveis e pontos de referência e, desta forma, o caminho particular de um estudante nada mais é do que uma questão de probabilidades e probabilidades esperadas. As trajetórias de aprendizado permitem especificar, em um nível adequado e mensurável de detalhes, quais ideias os estudantes precisam conhecer durante o desenvolvimento e a evolução de um dado conceito ao longo do tempo (CONFREY, 2012).

Por outro lado, o uso de linguagens de programação visuais (LPV) é explorado em várias áreas do conhecimento, tendo seu alicerce mais evidente no ensino de programação e em ferramentas para geração de código em determinadas tecnologias e frameworks.

Dentre tais estruturas, (DA SILVEIRA JÚNIOR et al., 2015; VANDEVELDE; VANDERBORGHT, 2013) citam o aumento na utilização de algumas $L P V s$ tais como o Scratch (MIT, 2018) e Google Blockly (GOOGLE, 2018), onde exploram sua aplicação na área de robótica e aprendizagem de algoritmos, tendo resultados interessantes quanto ao desempenho dos estudantes quando submetidos ao uso de tais tecnologias.

Cabe citar ainda o esforço de pesquisa no sentido de criar instrumentos que possam automatizar o processo de criação de trajetórias e recursos educacionais, conforme mostram (CANTO FILHO, A. B.; LIMA, J. V.; TAROUCO, 2017) ao propor um modelo de representação para recursos educacionais utilizando mapas conceituais.

No entanto, fica evidenciado a necessidade de pesquisas e iniciativas no sentido de explorar instrumentos e técnicas que possam aperfeiçoar a etapa de projeto para criação de trajetórias de aprendizagem, sobretudo soluções que possibilitem que o esforço desprendido para sua criação esteja direcionado para a concepção do percurso pedagógico, e não somente nos processos tecnológicos.

Neste sentido, esta pesquisa tem objetivo apresentar uma proposta de estrutura gráfica baseada nos princípios de design dos componentes de programação visual em blocos para projetar trajetórias de aprendizagem de forma iterativa.

Tal abordagem permite inúmeras aplicações na área de tecnologias educacionais, uma vez que ao estruturar uma trajetória na forma de blocos inter-relacionados é possível realizar ações de validação, análise e geração de outras estruturas, tais como soluções web e aplicativos para dispositivos móveis.

Faz parte também do escopo desta pesquisa apresentar os resultados de um estudo de caso aplicado com 27 estudantes de curso de Informática na Universidade Federal do Rio Grande do Sul (UFRGS), onde foram convidados a ordenar trajetórias de aprendizagem utilizando ferramenta desenvolvida no escopo desta pesquisa. Os resultados sugerem que o modelo consegue representar conceitos em uma trajetória com um nível de detalhamento bastante alto se comparado a outras técnicas, além de identificar que ao montar trajetórias na forma de blocos os estudantes apresentam 
progresso no desempenho quanto à assimilação do conteúdo sendo explorado, tornando a metodologia promissora também quanto ao aspecto do ensino e aprendizagem.

\section{Fundamentação teórica e trabalhos relacionados}

Este tópico apresenta a fundamentação teórica e trabalhos relacionados, onde são dispostas citações referentes à: 1) trajetórias de aprendizagem, mostrando suas diferentes definições; 2) estruturas gráficas para trajetórias de aprendizagem, ilustrando os esforços da literatura para sua representação e 3) linguagens de programação baseadas em blocos, evidenciando sua evolução nos últimos anos.

As pesquisas de (SIMON, 1995) introduziram a expressão "Trajetória Hipotética de Aprendizagem" para representar os caminhos pelos quais o aprendizado pode prosseguir quando os alunos progridem de um ponto de partida para um objetivo de aprendizagem pretendido. Representando uma progressão da cognição que, embora não necessariamente linear, também não é aleatório (SZTAJN et al., 2012).

Não obstante, o processo de análise do percurso pedagógico percorrido pelo aluno durante uma intervenção didática é uma prática encarada como a rota de aprendizagem que o aluno trilha, com o objetivo de atingir o conhecimento necessário acerca de um ou vários conceitos interligados a um tópico geral (NUNES et al., 2017).

Por outro lado, (CANTO FILHO, A. B.; LIMA, J. V.; TAROUCO, 2017) destacam a importância do uso de estruturas gráficas para representar recursos educacionais, onde usam uma notação baseada em mapas conceituais, conforme ilustra Figura 01, afirmando ser possível identificar conceitos e proposições presumidamente conhecidos pelo estudante que se relacionem com estudo da área de Física, explorando as possibilidades de modelagem e construção de objetos de aprendizagem.

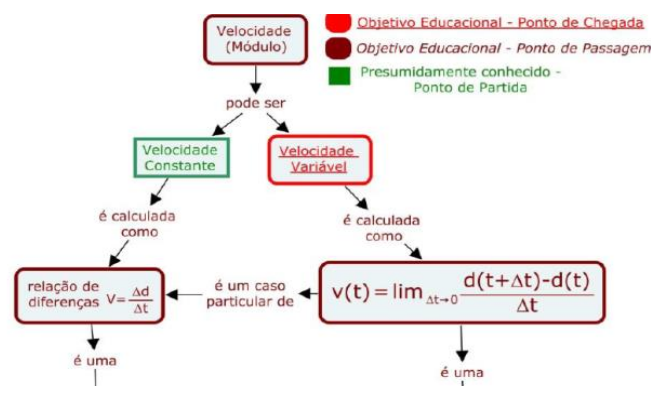

Figura 01 - Modelagem com mapas conceituais (CANTO FILHO, A. B. ; LIMA, J. V.; TAROUCO, 2017).

Por outro lado, (CONFREY, 2012) propõe realizar um mapeamento de trajetórias de aprendizagem do currículo de matemática de escolas Norte Americanas utilizando design baseado no uso de formas hexagonais, como é possível observar na Figura 02, destacando a dependência entre os conceitos com mapeamento por cores. 


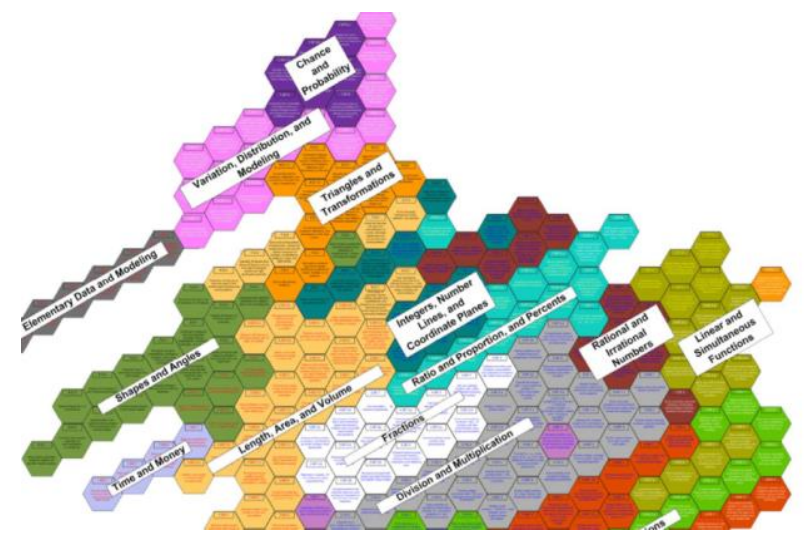

Figura 02 - Modelagem utilizando formas hexagonais (CONFREY, 2012).

Não obstante, (SIMBINE; LIMA, 2016) propõe um modelo baseado no uso de estruturas gráficas com eixos cartesianos para visualização interativa das trajetórias de aprendizagem, sendo tal modelo, segundo o autor, um instrumento capaz de simplificar a análise visual das possíveis atividades a serem executadas.

Já uma Linguagem de Programação Visual (LPV) baseada em blocos, segundo (VARELA, 2014), é um instrumento que permite ao usuário criar códigos de software através de manipulação gráfica, podendo arrastar e organizar logicamente blocos em uma tela de trabalho.

O uso de LPV vem sendo utilizado em várias áreas do saber, sobretudo como instrumento de aprendizagem, conforme citam (SUSILO et al., 2016), quando explora seu uso no ensino de robótica, (BRUMBAUGH; BRAMBAUGH; ROCK, 2006) ao estruturar jogos da área de matemática usando blocos e (BARATÈ et al., 2017) ao apresentar uma experiência educacional para o ensino de música em escolas no ensino secundário.

\section{Metodologia}

Tendo em vista a evidente existência de abordagens distintas para uso do termo "trajetórias de aprendizagem", conforme citam (LIMA et al., 2016), esta pesquisa propõe a adoção do conceito de Trajetórias de Aprendizagem Hipotética (TAH), inicialmente proposta por (SIMON, 1995), além de entendê-la como sendo um "caminho através de um corredor conceitual”, conforme visão de (CONFREY, 2012).

Já com relação à representação gráfica, adotou-se os princípios de design na forma de blocos, detalhado por (FRASER, 2015), onde ilustra padrões e boas práticas para uma linguagem de programação visual baseada em blocos.

Não obstante, para geração das estruturas gráficas foi utilizado o framework baseado na web denominado Google Blockly (GOOGLE, 2018), disponibilizando o arcabouço necessário para desenho e estruturação de novas formas geométricas baseadas em blocos, sendo que, para esta pesquisa, serão adotadas as boas práticas definidas por (PASTERNAK; FENICHEL; MARSHALL, 2017).

Por outro lado, o estudo de caso proposto foi realizado com encontros presenciais em laboratório, onde os participantes (27 estudantes de curso de informática 
da UFRGS) foram convidados a utilizar uma ferramenta web, desenvolvida no escopo desta pesquisa, para estruturar trajetórias de aprendizagem utilizando uma notação por blocos.

Para tanto, durante os encontros presenciais os alunos foram organizados em dois grupos: ao primeiro grupo foi designado o assunto "Introdução a Canvas com HTML5" e para o segundo adotou-se os conceitos de "Introdução ao CSS", assuntos normalmente tratados nos cursos de computação. Em etapa posterior os grupos foram invertidos, de tal forma que todos os alunos tiveram oportunidade de trabalhar os dois conteúdos.

Para esta pesquisa foi considerada uma abordagem metodológica quantitativa, com a aplicação de questionários de avaliação para mensurar a evolução dos alunos envolvidos no processo. Para isso, adotou-se método proposto por (DA SILVA; LOPES; JUNIOR, 2014), com aplicação de pré e pós-testes.

Neste cenário, o pré-teste teve objetivo de avaliar o conhecimento prévio dos alunos nos assuntos, sendo aplicado a todos os alunos participantes, sendo utilizado a ferramenta Google Forms para sua efetivação. Já para verificar a situação final com relação à assimilação do conteúdo, foi aplicado um pós-teste utilizando os mesmos processos e ferramentas de aplicação.

Ambos os questionários, pré e pós-testes, continham o mesmo número de questões e com nível de conhecimento equivalente, sugerindo um mecanismo de avaliação que permite identificar ao final do processo a evolução dos estudantes nos assuntos propostos.

\section{Resultados e discussão}

Conforme mencionado anteriormente, esta pesquisa propõe apresentar um modelo gráfico para representar trajetórias de aprendizagem, assim, tal estrutura, deve permitir a representação de conceitos, e garantir instrumentos para relacioná-los, ao mesmo tempo possuir formas de organizar assuntos por proximidade, em virtude da relação entre os assuntos. Não obstante, cabe à estrutura gráfica permitir o reuso dos componentes existentes para aplicações diversas.

Neste cenário, tal notação de representação baseia-se em estruturas retangulares na forma de blocos com pontos de encaixe, seja na horizontal, vertical ou em ambas as direções, conforme é possível observar na Figura 03.
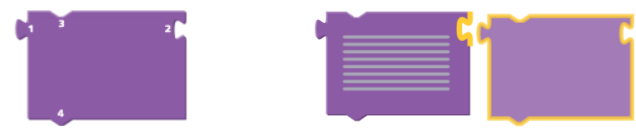

Figura 03 - Representação de conceitos em forma de blocos.

As formas ilustradas na Figura 03 indicam possibilidades de representação de conceitos em uma trajetória de aprendizagem, onde no escopo desta pesquisa serão denominados "Blocos de Conceito", sendo que o primeiro Bloco representa um conceito isolado, já o segundo elemento ilustra a possibilidade de agregação entre dois conceitos, permitindo a montagem de estruturas de forma iterativa. 
Ainda na Figura 03 é possível observar a presença de marcações numéricas nos elementos de vinculação. As conexões marcadas com 1 (um) e 2 (dois) indicam a possibilidade de conexão na horizontal, já as conexões 3 (três) e 4 (quatro) permitem relação na vertical entre conceitos. Cabe destacar ainda que é possível considerar variações deste modelo, permitindo conexões somente na horizontal ou vertical, de acordo com os objetivos pedagógicos a serem alcançados com a montagem das trajetórias de aprendizagem.

Neste sentido, partindo da possibilidade de relacionar blocos, a Figura 04 mostra representação de alguns agrupamentos de conceitos, sendo estruturadas utilizando a notação por blocos, onde cada macro conceito é representado por uma cor sólida aleatória e de um valor número ilustrativo para facilitar a visualização.

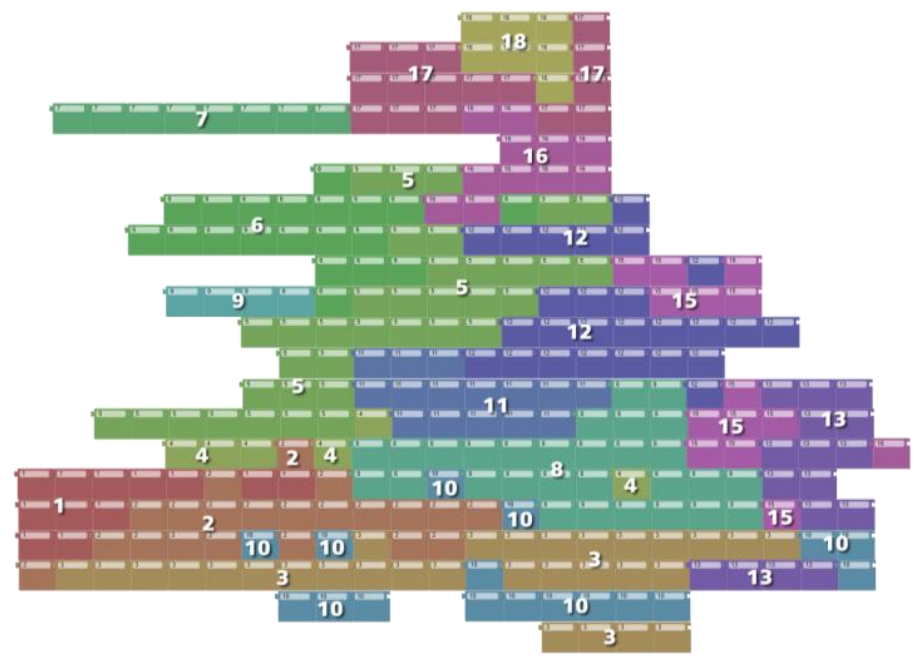

Figura 04 - Representação de 18 agrupamentos de Conceito usando notação por blocos

A representação da Figura 04 busca reproduzir uma proposta de organização de currículo proposto por (CONFREY; MALONEY; CORLEY, 2014), ao qual encara tal estrutura como sendo representações de trajetórias de aprendizagem, apresentando a evolução de uma grade curricular completa de um curso de Matemática de várias séries do ensino secundário de escolas Norte Americanas. Tal reprodução indica, visualmente, que o uso de blocos apresenta potencial no sentido de representar estruturas complexas na forma de relação entre conceitos.

Tal abordagem apresenta algumas características que podem ser consideradas promissoras, dentre elas é possível citar: 1) A utilização de estrutura de blocos. Conceito largamente difundido na última década, com tecnologias e bibliotecas que podem ser adaptadas para o contexto de design de trajetórias de aprendizagem; 2) Existência de frameworks robustos disponíveis com licença aberta para uso; 3) Linguagem de fácil entendimento e aplicação; 4) Possibilidade de agregar novos elementos para novas representações; 5) Garantia de reuso dos elementos gráficos em ambiente computacional; 6) Possibilidade de monitorar a execução do roteiro de aprendizagem; 7) Facilidade para criar mecanismos de iteração nos blocos, a fim de gerar conteúdo a partir da estruturação das trajetórias.

Por outro lado, o estudo de caso teve objetivo inicial de avaliar a viabilidade do uso da abordagem por blocos para representação de conceitos. Para tanto, os 
participantes foram submetidos ao uso de uma ferramenta $W e b$, desenvolvida no escopo desta pesquisa, conforme ilustra a Figura 05.

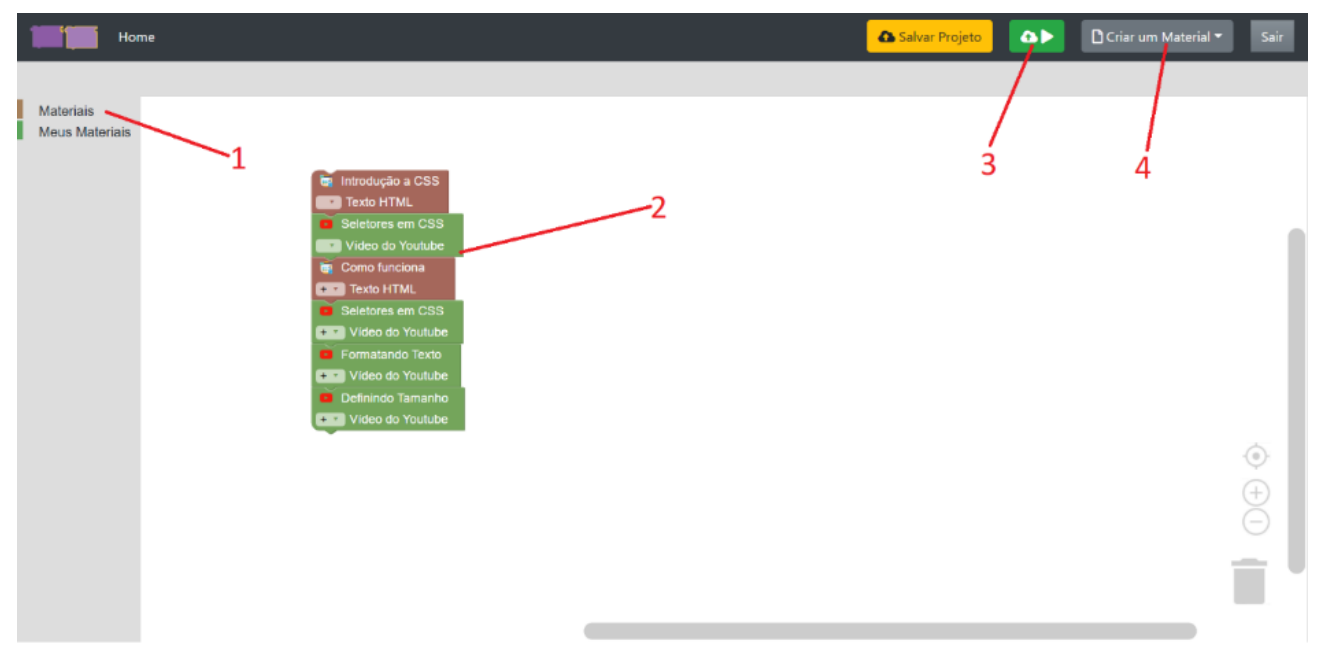

Figura 05 - Ferramenta web para criação de blocos de conceito

As marcações numéricas presentes na figura 05 ilustram a distribuição de funcionalidades da ferramenta, onde observamos: 1) Escolha do tipo de material, sendo possível optar por materiais disponibilizados pelo professor ou criados pelo aluno. 2) Área que permite agrupar os conceitos na forma de blocos. 3) Botão de execução, permitindo visualizar o agrupamento na forma de curso. 4) Opção para criação de novos materiais pelo aluno.

Ainda na Figura 05 a marcação numérica 2 (dois) mostra uma sequência de blocos conectados, onde cada bloco representa um conceito vinculado a um recurso educacional (texto, vídeo, simulador, $p d f$, etc.). Desta forma, é possível perceber que o primeiro elemento da sequência é um texto no formato $H T M L$, já o segundo bloco representa um vídeo do Youtube, sugerindo, desta forma, que ao conectar os conceitos é estruturado também uma rota pedagógica, com assuntos encadeados.

Para esta pesquisa optou-se por utilização de blocos de conceitos com encaixe somente na vertical, com intuito de permitir a percepção da sequencialidade dos assuntos. Cabe citar ainda, que a ferramenta desenvolvida permite a visualização do material didático vinculado a cada conceito no momento de realizar a relação entre os blocos, garantindo a leitura do conteúdo além da ação de criação da trajetória de aprendizagem.

O objetivo inicial da pesquisa foi avaliar como os participantes estruturam as trajetórias de forma livre, neste sentido, os 27 estudantes criaram um total de 50 diferentes trajetos para os dois assuntos propostos (Introdução a Canvas e Introdução a $C S S$ ) utilizando a notação por blocos de conceito. Neste cenário, foram utilizados 515 blocos, indicando uma média de 19,07 conceitos por cada participante, indicando que o modelo consegue representar diferentes visões para um mesmo assunto, fator que o torna promissor quanto modelo de representação. Além disso, 32\% dos materiais didáticos (texto $h t m l$, vídeos, $p d f$, etc.) utilizados foram cadastrados pelos próprios participantes da pesquisa (alguns materiais foram previamente disponibilizados, onde os 
participantes poderiam optar pelos materiais já existentes ou criar novos para montagem das trajetórias).
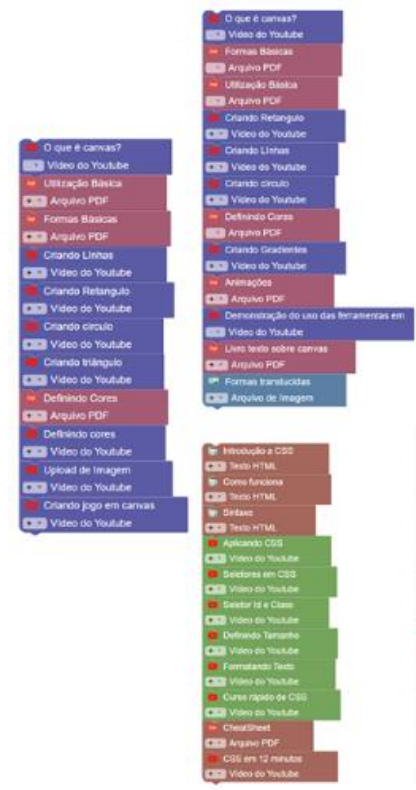
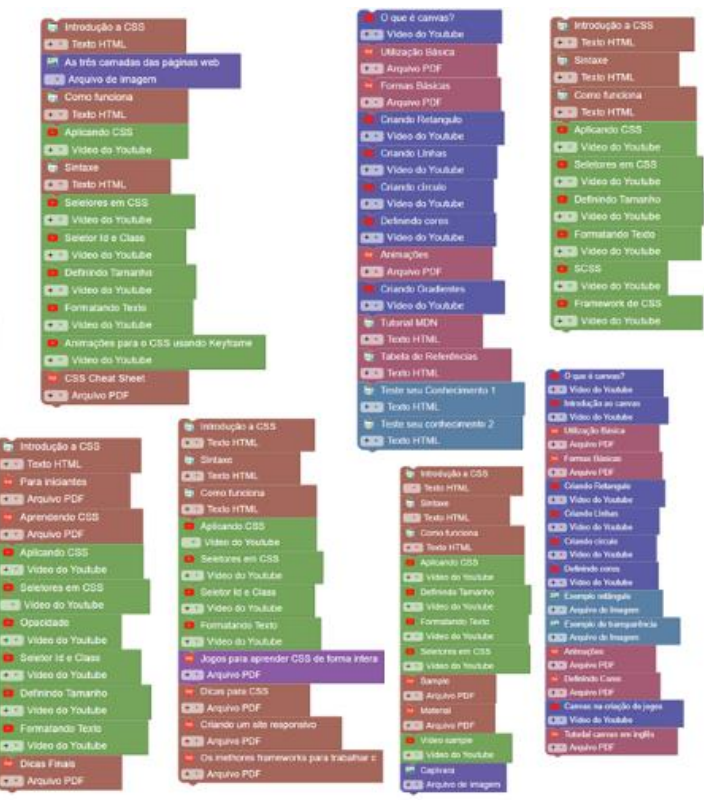

Figura 06 - Trajetórias montadas por participantes da pesquisa com utilização de notação por blocos.

A Figura 06 mostra algumas das 50 trajetórias montadas por participantes do experimento, onde podemos observar a presença de blocos distintos para os dois assuntos propostos na pesquisa. Observa-se nesta figura a variedade de trajetórias criadas, demonstrando potencial do instrumento para aplicações futuras com exploração de outros aspectos do processo ensino e aprendizagem.

Por outro lado, os participantes foram submetidos a um teste antes da montagem das trajetórias e após o processo. Tal abordagem metodológica foi adotada partindo da hipótese que ao montar as trajetórias na forma de blocos os estudantes teriam uma melhor compreensão dos assuntos propostos, uma vez que teriam que realizar uma apropriação dos conteúdos para identificar a melhor forma de relacioná-los.

Neste sentido, os dados de desempenho indicam que houve uma melhora quanto a média obtida pelos os estudantes dos dois grupos (Grupo A: Introdução Canvas e Grupo B: Introdução a CSS), além de evolução quanto a homogeneidade da turma, conforme indica o desvio padrão constante na Tabela 01. Além disso, o delta médio em cada grupo confirma a evolução por aluno na assimilação do conteúdo.

\begin{tabular}{|l|l|l|}
\hline Grupo & Pré-Teste & Pós-Teste \\
\hline Grupo A & Média: 7,99 & Média: 8,68 \\
- Delta médio: 0,74 & Desvio Padrão: 1,43 & Desvio Padrão: 1,29 \\
\hline Grupo B & Média: 7,07 & Média: 8,62 \\
- Delta médio: 1,56 & Desvio Padrão: 2,60 & Desvio Padrão: 1,23 \\
\hline
\end{tabular}

Tabela 01. Desempenho de participantes da pesquisa.

Além disso, o Gráfico 01 apresenta a dispersão dos participantes no pré e pósteste, indicando que após a utilização do modelo proposto os participantes ficam mais concentrados próximo a média, sugerindo uma certa evolução quanto a homogeneidade. 

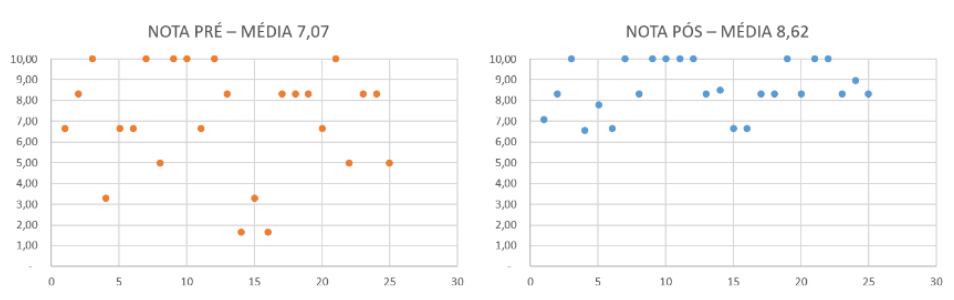

Gráfico 01. Dispersão dos participantes do Grupo B

\section{Considerações finais}

Esta pesquisa teve como objetivo apresentar um modelo de representação gráfica que permita projetar trajetórias de aprendizagem, em um contexto onde tais trajetórias também podem ser encaradas como uma progressão em determinado conteúdo.

Os esforços existentes na literatura para propor soluções para projeto de trajetórias de aprendizagem reforçam que a solução aqui apresentada se mostra potencialmente promissora por uma série de aspectos intrínsecos inerentes às tecnologias e métodos adotados.

Por outro lado, o estudo de caso apresentado evidencia que o uso das ferramentas e métodos indicam um caminho no sentido de sua utilização também no processo de ensino e aprendizagem, conforme a evolução no desempenho observados na pesquisa.

Por fim, entende-se ainda a necessidade de esforços complementares, na forma de trabalhos futuros, sobretudo nos seguintes temas: a) Explorar outros elementos para design de blocos.; b) Aplicação com professores e estudantes levando em conta os aspectos de confecção e execução das trajetórias, além de aumentar a amostra; c) Melhorar os aspectos de interface e usabilidade; d) Incluir questionários na plataforma $w e b ;$ e) Avaliar potencial de geração de conteúdo para ambientes mobile utilizando o conteúdo criado por blocos; f) Incluir recursos para classificação por dificuldade de conceito; g) Explorar aspecto do tempo de utilização de cada elemento na plataforma.

\section{Referências Bibliográficas}

BARATÈ, A. et al. Fostering Computational Thinking in Secondary School through Music - An Educational Experience based on Google Blockly. Proceedings of the 9th International Conference on Computer Supported Education.

Anais...2017Disponível em:

$<$ http://www.scitepress.org/DigitalLibrary/Link.aspx?doi=10.5220/0006313001170124 $>$

BORBA, M. DE C. Informática e Educação Matemática. 5. ed. Belo Horizonte: Autêntica Editora, 2016.

BRUMBAUGH, D. K.; BRAMBAUGH, L.; ROCK, D. Scratch your brain geometry: Math Games, Tricks, and Quick Activities. [s.1.] Critical Thinking Company, 2006.

CANTO FILHO, A. B. ; LIMA, J. V. ; TAROUCO, L. M. R. Mapas Conceituais de Projeto: uma ferramenta para projetar objetos de aprendizagem significativa. Ciência \& Educação (Bauru), v. 23, n. 3, p. 723-740, 2017. 
CONFREY, J. Articulating a Learning Sciences Foundation for Learning Trajectories in the CCSS-M. PMENA, 2012.

CONFREY, J.; MALONEY, A. P.; CORLEY, A. K. Learning trajectories: a framework for connecting standards with curriculum. ZDM - International Journal on Mathematics Education, v. 46, n. 5, p. 719-733, 2014.

DA SILVA, D.; LOPES, E. L.; JUNIOR, S. S. B. Pesquisa Quantitativa: Elementos, Paradigmas e Definições. Revista de Gestão e Secretariado, v. 05, n. 01, p. 01-18, 2014.

DA SILVEIRA JÚNIOR, G. et al. Análise da ferramenta de programação visual blockly como recurso educacional no ensino de programação. III Seminário Argentina-Brasil de Tecnologias da Informação e da Comunicação. Anais...2015

FRASER, N. Ten things we've learned from Blockly. Proceedings - 2015 IEEE Blocks and Beyond Workshop, Blocks and Beyond 2015. Anais...2015

GOOGLE. Google Blockly. Disponível em: 〈https://developers.google.com/blockly/〉. Acesso em: 17 abr. 2018.

LIMA, J. V. DE et al. Trajetórias de Aprendizagem: Teoria e Prática. [s.1.] Createspace Independent Pub, 2016.

MIT. Scratch - imagine, program, share. Disponível em: <https://scratch.mit.edu>. Acesso em: 17 abr. 2018.

NUNES, F. B. et al. Análise e Recomendação de trajetos de aprendizagem em mundos virtuais. Revista Novas Tecnologias na Educação (RENOTE), v. 15, n. 1, 2017.

PASTERNAK, E.; FENICHEL, R.; MARSHALL, A. N. Tips for Creating a Block Language with Blockly. 2017 IEEE Blocks and Beyond Workshop, 2017.

PELIZZARI, A. et al. Teoria da aprendizagem significativa segundo Ausubel. Revista PEC, v. 2, n. 1, p. 37-42, 2002.

RESNICK, M. et al. Scratch: Programming for All. Communications of the ACM, v. 52, p. 60-67, 2009.

SIMBINE, F. B.; LIMA, J. V. DE. Visualização Interativa das Trajetórias de Aprendizagem. Porto Alegre: CINTED, 2016

SIMON, M. A. Reconstructing Mathematics Pedagogy from a Constructivist Perspective. Journal for Research in Mathematics Education, v. 26, n. 2, p. 114, 1995.

SUSILO, E. et al. STORMLab for STEM Education: An Affordable Modular Robotic Kit for Integrated Science, Technology, Engineering, and Math Education. IEEE Robotics and Automation Magazine, v. 23, n. 2, p. 47-55, 2016.

SZTAJN, P. et al. Learning Trajectory Based Instruction: Toward a Theory of Teaching. Educational Researcher, v. 41, n. 5, p. 147-156, 2012.

VANDEVELDE, C.; VANDERBORGHT, B. Overview of Technologies for Building Robots in the Classroom. International Conference on Robotics in Education, Proceedings, p. 122-130, 2013. 
VARELA, H. Aprenda a programar com scratch: Uma introdução visual à programação com jogos, arte, ciência e matemática. 1. ed. São Paulo: Novatec Editora, 2014.

ZUNGUZE, M. C. et al. Relação entre Estilos de Aprendizagem e forma de navegação em Apresentações Paralelas Multimídia Relationship between Learning Styles and Way of Navigation in Parallel Presentations Multimedia. Informática na educação: teoria \& prática, p. 15-26, 2017. 